\title{
USO DE BLOGS Y EVALUACIÓN CONTINUA DEL APRENDIZAJE DEL ALUMNADO UNIVERSITARIO
}

\section{THE USE OF BLOGS AND THE CONTINUOUS ASSESSMENT OF UNIVERSITY STUDENT LEARNING}

\author{
Pere Molina; juan.p.molina@uv.es \\ Luís Antolín Jimeno; luis.antolin@uv.es \\ Víctor Pérez-Samaniego; victor.m.perez@uv.es \\ José Devís-Devis; jose.devis@uv.es \\ Miguel Villamón; miquel.villamon@uv.es \\ Universitat de València \\ Javier Valenciano Valcárcel; javier.valenciano@uclm.es \\ Universidad de Castilla La Mancha
}

\section{RESUMEN:}

Se presenta una experiencia de innovación educativa consistente en la evaluación continua del aprendizaje mediante el uso de blogs. Se desarrolló durante el curso 2010-2011, en un grupo de cinco asignaturas de las Universidades de Valencia y Castilla La Mancha. Las diversas formas de evaluación del aprendizaje que se implementaron, se concretan en tres tipos de usos participativos de los blogs: a) a través de los comentarios; b) de la elaboración de posts; o, c) como administradores. La experiencia llevada a cabo muestra que los blogs pueden ser un valioso instrumento para promover el trabajo autónomo de los estudiantes y contribuir a la actualización de las formas de evaluación tradicionales.

PALABRAS CLAVE: Evaluación continua, blog, enseñanza no presencial, e-learning.

\begin{abstract}
:
This paper presents the experience of an innovative educational project on continuous assessment of students' learning using blogs implemented by a group of teachers at the University of Valencia and the University of Castilla La Mancha during the 2010-11 academic year. Three types of assessment were implemented by the use of interactive blogs: a) using comments; b) through posts; c) joining blogs as administrators. The experience shows the potential of blogs as an instrument for autonomous work of students and the updating of traditional forms of assessment.
\end{abstract}

KEYWORDS: Continuous assessment, blog, distance learning, e-learning. 


\section{INTRODUCCIÓN}

Los blogs son sitios web donde uno o varios autores publican periódicamente mensajes (también llamados entradas o posts) con información, generalmente textual, pudiendo sus lectores participar añadiendo comentarios. Los mensajes aparecen organizados cronológicamente, pero de forma inversa, es decir, mostrando en primer lugar la entrada más reciente. La popularización de estos sitios web, la sencillez de manejo y la posibilidad de incorporar contenidos multimedia o enlazar otras fuentes de información relacionadas, acerca su uso a un gran número de personas. Pero es la rapidez en la interacción y la facilidad de participación, las dos características clave que las diferencia de otras herramientas virtuales y le confiere mayores posibilidades didácticas (Stefanac, 2006; Bohórquez, 2008; González Sánchez y García Muiña, 2009).

Los blogs educativos o edublogs pueden ser un recurso importante en el marco del Espacio Europeo de Educación Superior (EEES) que enfatiza el aprendizaje por encima de la enseñanza. Su uso implica nuevas habilidades que conectan con la búsqueda y generación de conocimiento, así como el trabajo colaborativo, ingredientes todos ellos de una valiosa experiencia de aprendizaje. Además, los blogs propician la interactividad entre los alumnos y les ayudan a explorar material adicional y complementario para la asignatura (Glogoff, 2005; Torres, 2009; Santoveña, 2011) y permiten prolongar las actividades formativas desarrolladas por los estudiantes universitarios más allá de las aulas, ampliando las oportunidades de aprendizaje a la vida cotidiana, de tal manera que pueda germinar en un aprendizaje para toda la vida (Gewerc, 2005; Ferreyro, 2007; Marzal y Butera, 2007).

Ante este potencial didáctico no es de extrañar que en los últimos años hayan aparecido publicadas numerosas experiencias e investigaciones, de carácter nacional e internacional, relacionadas con distintas dimensiones o aspectos vinculados al uso de blogs en la educación universitaria. Entre los temas tratados encontramos: las opiniones, motivaciones y satisfacción de los estudiantes en el uso de los blogs (Lorenzo, Trujillo, Lorenzo y Pérez. 2011; Deng y Yuen, 2012; Kerawalla, Minocha, Kirkup y Conole, 2008; Wang, Lin y Liao, 2012), el uso de blogs para estimular las prácticas reflexivas entre los estudiantes universitarios (Hernández-Ramos, 2004; Ladyshewsky y Gardner, 2008; Woperies, Sloep y Portman, 2010; Xie, Ke y Sharma, 2008), la evaluación global de proyectos o iniciativas alrededor de la creación y uso de blogs (Farmer, Yue y Brooks, 2008; Orihuela y Santos, 2004) y las percepciones de los estudiantes sobre los blogs y su uso en el proceso de aprendizaje (Aguaded, López y Alonso, 2009; Dickey, 2004; Pérez-Nevado, Aranda, Hernández, Martín, Benito y Córdoba, 2012; Salinas y Viticcioli, 2008; Shim y Guo, 2009;). Otras investigaciones se ocupan de aspectos concretos del contenido disciplinar de una materia (Ducate y Lomicka, 2008; Phillip y Nichols, 2009) u otros aspectos más puntuales como el análisis y clasificación de blogs según los principios de las buenas prácticas (González Sánchez y García Muiña, 2009).

El uso de blogs en el contexto de la enseñanza universitaria admite tal variedad de investigaciones posibles como cualquier otro recurso, metodología o proyecto educativo. Pero en el contexto del EEES cobra especial interés la repercusión que pueda tener en el aprendizaje de los estudiantes $y$, por extensión, a las formas de evaluación de dichos aprendizajes. Por ello el objetivo de este trabajo es presentar los resultados de un proyecto de innovación educativa, basado en la evaluación continua del aprendizaje del alumnado universitario, a través de su participación en la utilización de blogs. 


\section{CARACTERÍSTICAS DEL PROYECTO}

El proyecto de innovación pretendía desarrollar formas de evaluación continua del aprendizaje del alumnado adecuadas a diversos usos de blogs, tal y como se hallan descritos en Antolín, Molina, Villamón, Devís y Pérez (2011). Los principales objetivos del proyecto eran:

- Definir formas de evaluación continua del aprendizaje del alumnado, según el tipo de uso y características del blog, en cada una de las asignaturas implicadas en el proyecto.

- Relacionar las formas de evaluación del aprendizaje definidas con el desarrollo de competencias vinculadas al análisis y tratamiento de la información, y con la utilización de las TIC por parte del alumnado.

- Establecer la relación entre la evaluación continua del aprendizaje del alumnado, con el trabajo no presencial determinado en los créditos ECTS.

- Desarrollar en la práctica los diversos tipos de evaluación continua del aprendizaje del alumnado.

El proyecto se desarrolló durante el curso 2010-2011 en cuatro asignaturas de la Facultad de Ciencias de la Actividad Física y el Deporte de la Universitat de València (UV) y una de la Facultad de Educación de Toledo de la Universidad de Castilla La Mancha (UCLM), cuyas características académicas se concretan en la tabla 1.

\begin{tabular}{|c|c|c|c|}
\hline Titulación y centro & Asignaturas & $\begin{array}{l}\text { Características académicas de } \\
\text { las asignaturas }\end{array}$ & $\begin{array}{c}\text { Grupos, número de } \\
\text { estudiantes y profesores al } \\
\text { cargo }\end{array}$ \\
\hline \multirow{2}{*}{$\begin{array}{l}\text { Licenciatura en } \\
\text { Ciencias de la } \\
\text { Actividad Física y el } \\
\text { Deporte de la UV }\end{array}$} & $\begin{array}{l}\text { Diseño curricular } \\
\text { de la educación } \\
\text { física }\end{array}$ & $\begin{array}{l}\text { Troncal cuatrimestral (1er } \\
\text { semestre) de } 2 \text { o ciclo ( } 5 \text { o } \\
\text { curso) de } 4^{\prime} 5 \text { créditos ( } 3 \\
\text { teóricos y } 1^{\prime} 5 \text { prácticos) }\end{array}$ & $\begin{array}{l}1 \text { grupo de teoría y } 4 \text { de } \\
\text { práctica con un total de } 133 \\
\text { estudiantes y } 1 \text { profesor }\end{array}$ \\
\hline & $\begin{array}{l}\text { Teoría y práctica } \\
\text { del curriculum de } \\
\text { la educación física }\end{array}$ & $\begin{array}{l}\text { Troncal anual de } 20 \text { ciclo ( } 4 \text { o } \\
\text { curso) de } 9 \text { créditos ( } 6 \\
\text { teóricos y } 3 \text { prácticos) }\end{array}$ & $\begin{array}{l}2 \text { grupos de teoría y } 6 \text { de } \\
\text { práctica con un total de } 196 \\
\text { estudiantes y } 2 \text { profesores }\end{array}$ \\
\hline \multirow{2}{*}{$\begin{array}{l}\text { Grado en Ciencias } \\
\text { de la Actividad } \\
\text { Física y el Deporte } \\
\text { de la UV }\end{array}$} & $\begin{array}{l}\text { El juego educativo } \\
\text { y la iniciación } \\
\text { deportiva }\end{array}$ & $\begin{array}{l}\text { Fundamental básica } \\
\text { cuatrimestral de } 2 \text { o curso de } 6 \\
\text { créditos ECTS }\end{array}$ & $\begin{array}{l}1 \text { grupo de teoría y } 2 \text { de } \\
\text { práctica con un total de } 81 \\
\text { estudiantes y } 1 \text { profesor }\end{array}$ \\
\hline & $\begin{array}{l}\text { Fundamentos de } \\
\text { la expresión } \\
\text { corporal }\end{array}$ & $\begin{array}{l}\text { Obligatoria cuatrimestral de } \\
\text { 20 curso de } 6 \text { créditos ECTS }\end{array}$ & $\begin{array}{l}2 \text { grupos de práctica con un } \\
\text { total de } 50 \text { estudiantes y } 1 \\
\text { profesor }\end{array}$ \\
\hline $\begin{array}{l}\text { Grado en Maestro } \\
\text { de Educación } \\
\text { Primaria de la } \\
\text { Facultad de } \\
\text { Educación de } \\
\text { Toledo de la UCLM }\end{array}$ & $\begin{array}{l}\text { La educación } \\
\text { física como } \\
\text { materia escolar }\end{array}$ & $\begin{array}{l}\text { Obligatoria cuatrimestral de } \\
\text { 1er curso de } 6 \text { créditos ECTS }\end{array}$ & $\begin{array}{l}3 \text { grupos con un total de } 180 \\
\text { estudiantes y } 1 \text { profesor }\end{array}$ \\
\hline
\end{tabular}

Tabla 1. Estudios y centros participantes, asignaturas, características académicas y grupos de estudiantes y profesores implicados en la experiencia. 


\section{METODOLOGÍA}

Antes del periodo lectivo de cada una de las asignaturas, los profesores diseñaron individualmente los blogs y las actividades para darles contenido, así como el modo en que serían integrados en el sistema de evaluación y en la guía docente de cada una de las asignaturas (ver figura 1). La naturaleza teórica y/o práctica de cada asignatura y la experiencia previa de cada profesor en el uso de los blogs, fueron los principales determinantes a la hora de concretar de qué manera se aplicaría la evaluación continua, durante el periodo lectivo de la asignatura.

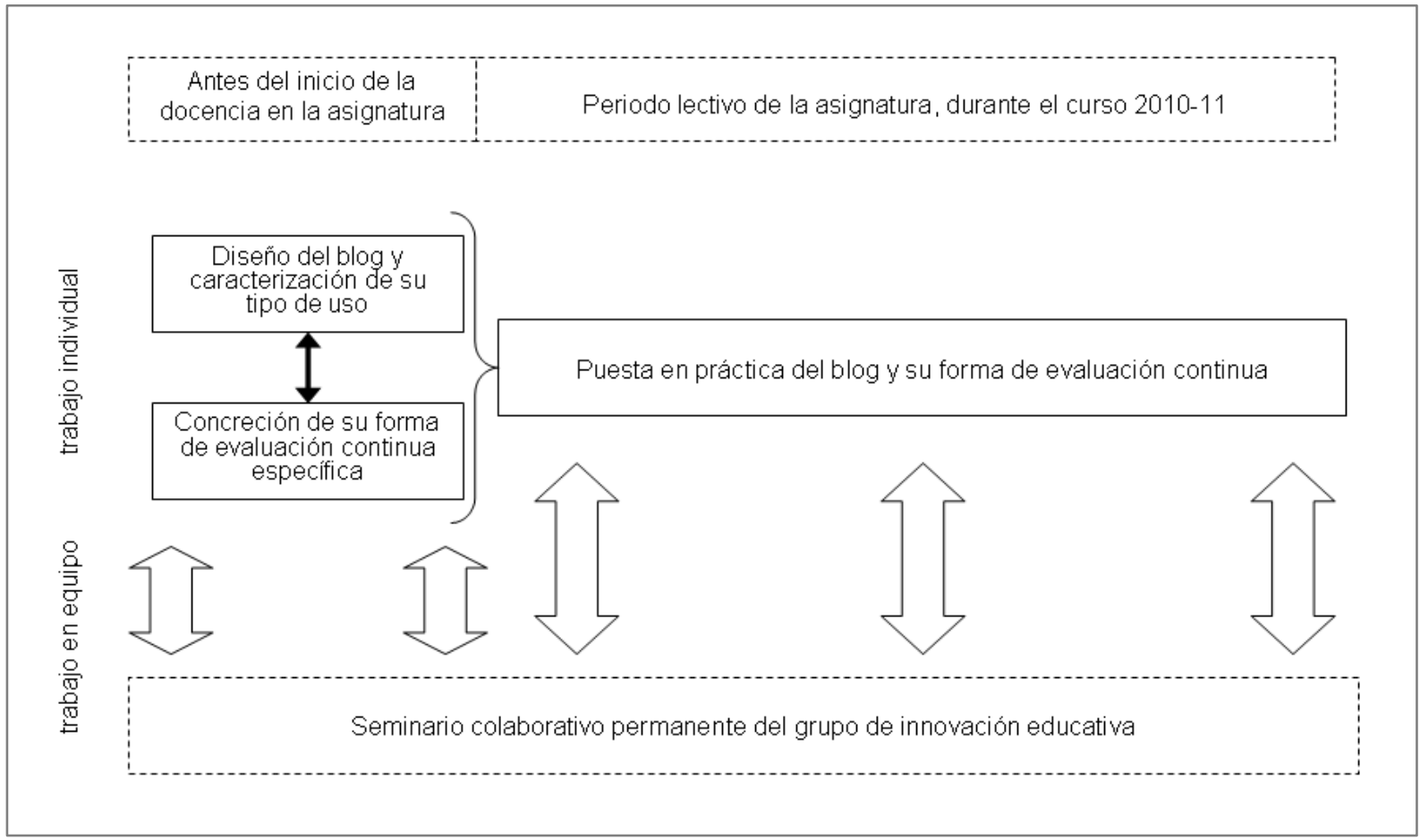

Figura 1. Proceso seguido

Para el seguimiento de la experiencia se recurrió al seminario colaborativo permanente, entendido como una técnica activa de enseñanza e investigación basada en el trabajo en grupo e intercambio de información, utilizada para profundizar desde el debate y análisis colectivo en un tema determinado (Fraile, 2002). En nuestro caso, se trataba de compartir las propuestas de acción evaluativa.

Cabe advertir que estamos ante un proyecto que lo que busca no es la recopilación de datos para su posterior análisis, sino que este análisis vaya parejo a la recopilación de los datos. No se pretende mejorar el proceso de enseñanza-aprendizaje al final de la experiencia, sino durante la misma. Por este motivo, la metodología seguida aunó los procesos de desarrollo y análisis de la experiencia. 


\section{DESCRIPCIÓN DE LA EXPERIENCIA}

\subsection{Estilos de uso de los blogs}

Las formas de evaluación desarrolladas tuvieron como referente cuatro estilos básicos del uso de los blogs, en función de la participación del alumnado, descritos en Antolín et al. (2011), que se definen y caracterizan de la siguiente forma:

- El blog docente como transmisor de información de la asignatura. Este uso se caracteriza por la utilización por parte del profesor del blog como un espacio virtual donde poder aportar a los estudiantes información relacionada con los contenidos o el desarrollo de la asignatura.

- El blog docente abierto a la participación del alumnado a través de sus comentarios. Este tipo de uso está caracterizado por la demanda de participación del alumnado a través de sus comentarios para la realización de actividades. Los estudiantes comentan los posts que elabora el profesor.

- El blog docente abierto a la participación del alumnado a través de sus entradas. Su característica es que, además de poder realizar comentarios a los posts elaborados por el profesor, los estudiantes también pueden elaborar y publicar sus posts. Los estudiantes colaboran como autores del blog.

- Los alumnos como administradores de sus blogs. En este uso los estudiantes se convierten en administradores de sus blogs, tomando decisiones sobre el contenido y la forma de los posts, el diseño y configuración del blog, etc.

En la tabla 2 se relacionan los blogs utilizados en las asignaturas participantes en el proyecto con los estilos básicos de uso descritos. Como el uso del blog docente como transmisor de la información, no está relacionado con la participación o interacción del alumnado en el blog, no se ha desarrollado un proceso de evaluación continua del aprendizaje en este estilo de uso.

\begin{tabular}{|c|c|c|}
\hline Asignatura & Blogs utilizados & Uso \\
\hline \multirow{2}{*}{$\begin{array}{l}\text { Diseño } \\
\text { curricular de } \\
\text { la educación } \\
\text { física }\end{array}$} & $\begin{array}{l}\text { Blog docente específico de la asignatura: } \\
\text { http://dissenyef2010.blogspot.com }\end{array}$ & $\begin{array}{l}\text { El blog docente abierto a la } \\
\text { participación del alumnado a } \\
\text { través de sus comentarios. }\end{array}$ \\
\hline & $\begin{array}{l}\text { Revista-blog complementaria y transversal: } \\
\text { http://didactica-afe.blogspot.com }\end{array}$ & $\begin{array}{l}\text { El blog docente abierto a la } \\
\text { participación del alumnado a } \\
\text { través de sus entradas. }\end{array}$ \\
\hline \multirow{3}{*}{$\begin{array}{l}\text { Teoría y } \\
\text { práctica del } \\
\text { currículum } \\
\text { de la } \\
\text { educación } \\
\text { física }\end{array}$} & $\begin{array}{l}\text { Blog docente específico de la asignatura: } \\
\underline{\text { http://tpcef.blogspot.com }}\end{array}$ & $\begin{array}{l}\text { El blog docente abierto a la } \\
\text { participación del alumnado a } \\
\text { través de sus comentarios. }\end{array}$ \\
\hline & $\begin{array}{l}\text { Revista-blog complementaria: } \\
\text { http://didactica-afe.blogspot.com }\end{array}$ & $\begin{array}{l}\text { El blog docente abierto a la } \\
\text { participación del alumnado a } \\
\text { través de sus entradas. }\end{array}$ \\
\hline & $\begin{array}{l}\text { Blogs individuales de alumnos: } \\
\text { http://samuellpezcarriltpcef.blogspot.com } \\
\text { http://pablotierrasecatpcef.blogspot.com } \\
\underline{\text { http://gemasanchistpcef.blogspot.com }} \\
\underline{\text { http://gonzalomonforttpcef.blogspot.com }}\end{array}$ & $\begin{array}{l}\text { Los alumnos como } \\
\text { administradores de sus } \\
\text { blogs. }\end{array}$ \\
\hline
\end{tabular}




\begin{tabular}{|c|c|c|}
\hline & http://manuelcarrilerotpcef.blogspot.com & \\
\hline $\begin{array}{l}\text { El juego } \\
\text { educativo y } \\
\text { la iniciación } \\
\text { deportiva }\end{array}$ & $\begin{array}{l}\text { Blogs de grupos de alumnos: } \\
\text { http://juniorfijo.blogspot.com/2011/05/introduccion- } \\
\text { somos-alumnos-de-segundo.html } \\
\text { http://juegosdeinvasion.wordpress.com } \\
\text { http://anbelmar.blogs.uv.es }\end{array}$ & $\begin{array}{l}\text { Los alumnos como } \\
\text { administradores de sus } \\
\text { blogs. }\end{array}$ \\
\hline $\begin{array}{l}\text { Fundamentos } \\
\text { de } \\
\text { expresión } \\
\text { corporal }\end{array}$ & $\begin{array}{l}\text { Blog docente específico de la asignatura } \\
\text { http://expresioncorporal1011.blogspot.com }\end{array}$ & $\begin{array}{l}\text { El blog docente abierto a la } \\
\text { participación del alumnado a } \\
\text { través de sus comentarios. }\end{array}$ \\
\hline $\begin{array}{l}\text { La educación } \\
\text { física como } \\
\text { materia } \\
\text { escolar }\end{array}$ & $\begin{array}{l}\text { Blog docente específico de la asignatura } \\
\text { http://efme2010.blogspot.com }\end{array}$ & $\begin{array}{l}\text { El blog docente abierto a la } \\
\text { participación del alumnado a } \\
\text { través de sus comentarios. }\end{array}$ \\
\hline
\end{tabular}

Tabla 2. Nombre de las asignaturas, blogs utilizados y tipología de uso ${ }^{1}$.

\subsection{Formas de evaluación continua desarrolladas}

A continuación se describen los usos concretos de los blogs y las formas de evaluación continua del aprendizaje del alumnado desarrolladas en cada una de las asignaturas objeto de la experiencia.

\section{a) Diseño curricular de la Educación Física}

Se utilizaron dos blogs: uno específico (http://dissenyef2010.blogspot.com) relacionado con el desarrollo de los contenidos de la asignatura y otro (http://didactica-afe.blogspot.com) en forma de revista como actividad complementaria y transversal de la asignatura. El blog específico se utilizó prioritariamente para presentar, al finalizar cada tema, las posibles preguntas de examen a las que el alumnado tenía que responder a partir de la lectura de los apuntes y el material bibliográfico del tema facilitados por el profesor a través del Aula Virtual y del blog. Para asegurar que el trabajo de los estudiantes fuese continuado, la posibilidad de realizar comentarios a los posts con posibles preguntas de examen estaba activa durante dos semanas. Terminado este plazo, se desactivaba esta opción y los estudiantes no podían introducir comentarios con sus respuestas.

La revista-blog fue una iniciativa de esta asignatura y de la de Teoría y práctica del currículum de la Educación Física, donde los estudiantes publicaban artículos relacionados con la enseñanza de las actividades físicas y deportivas, una vez eran evaluados positivamente por un consejo de redacción integrado por compañeros y compañeras de ambas asignaturas.

Un 20\% de la calificación de los estudiantes en esta asignatura correspondió a su participación en ambos blogs. Se realizó atendiendo criterios cuantitativos como: 1) la realización de 12 comentarios en el blog específico donde, al menos, se respondiese a una pregunta por tema; y 2) la publicación de un post (podía realizarse en grupo), realizar 3 comentarios o participar en la evaluación de artículos como miembro del consejo de redacción. Así como criterios cualitativos como: 1) la corrección ortográfica y gramatical en

\footnotetext{
${ }^{1}$ Algún blog puede tener su acceso restringido finalizado el curso académico 2010-11.
} 
el uso de la lengua (sea la castellana o la valenciana); 2) no cortar y pegar información de los apuntes, ni reproducir información ya escrita por los compañeros, sino debatirla, ampliarla, matizarla o corregirla desde el punto de vista del estudiante; 3) aportar opinión personal fundamentada en referencia bibliográficas de los temas; y 4) valorar especialmente los posts publicados en la revista-blog.

El proceso de evaluación continua en relación con la participación en los blogs también integró dos posts: uno dedicado a la realización, por parte de los estudiantes, de una valoración del blog específico de la asignatura (http://dissenyef2010.blogspot.com/2010/12/questio-36-tema-8.html) y otro que consistió en una autoevaluación del alumnado referida a su participación en los dos blogs utilizados en la asignatura (http://dissenyef2010.blogspot.com/2010/12/questio-37-tema-8.html). El proceso de evaluación continua se cerraba con una pequeña entrevista que mantuvo el profesor con cada uno de los estudiantes donde se hacía referencia a su participación concreta en los blogs.

\section{b) Teoría y práctica del currículum de la Educación Física}

En esta asignatura se utilizó un blog específico de la asignatura (http://tpcef.blogspot.com/) que ya había estado activo en otro curso académico, y que servía fundamentalmente para proporcionar información, materiales y presentar actividades. Los estudiantes podían dejar sus comentarios en cada una de las entradas y desde este blog acceder a otros administrados por los estudiantes que seguían los contenidos de la asignatura (por ejemplo: http://samuellpezcarriltpcef.blogspot.com, http://pablotierrasecatpcef.blogspot.com, http://gemasanchistpcef.blogspot.com, http://gonzalomonforttpcef.blogspot.com, http://manuelcarrilerotpcef.blogspot.com). En esta asignatura también se utilizó la revistablog, mencionada anteriormente (http://didactica-afe.blogspot.com). Los blogs personales de los estudiantes y las aportaciones a la revista-blog se tuvieron en cuenta para evaluar la asignatura en un $40 \%$ sobre el total de la calificación de los estudiantes.

El blog de la asignatura evolucionó de formas de participación más controladas por el profesor a otras más abiertas al control de los propios estudiantes. Sin dejar de perder su función informativa, el blog de la asignatura pasó a ganar peso como elemento dinamizador de las aportaciones de los estudiantes. A nivel técnico, la visualización automática en el blog de la asignatura de las actualizaciones de los blogs personales de los estudiantes se convirtió en una herramienta fundamental para favorecer la participación y la interacción entre los estudiantes entre sí y con los profesores, así como para facilitar un seguimiento continuado de sus aportaciones.

El resultado es que el aprendizaje se fue distribuyendo y diversificando en los distintos blogs personales de los estudiantes que, a su vez, creaban sus propias comunidades de seguidores. La principal consecuencia para la evaluación de este tránsito o evolución hacia la creación de una comunidad de aprendizaje fue la emergencia de la evaluación horizontal, entre pares o peer to peer. La siguiente entrada, en la que los propios estudiantes recomiendan visitar blogs de sus compañeros, resulta un ejemplo ilustrativo de desarrollo de la evaluación horizontal (http://tpcef.blogspot.com/2010/11/recomendaciones-blogueras.html). 


\section{c) El juego educativo y la iniciación deportiva}

Un 45\% de la evaluación del aprendizaje del alumnado en esta asignatura se dedicó a la elaboración de trabajos por el alumnado. Dos terceras partes de este porcentaje tenían una vinculación con el uso de blogs por parte del alumnado.

La dinámica de trabajo era la siguiente. El alumnado conformaba grupos reducidos de trabajo (3-4 estudiantes). Elegía un contenido de los presentados por el profesor sobre juegos elaboraban una sesión, la llevaban a la práctica en clase y luego daban forma escrita al tema (incluyendo la sesión) que se presentaba en forma de blog (pudiendo utilizar todos los recursos y herramientas complementarias del blog). El profesor evaluaba el desarrollo de la sesión en la práctica y el trabajo escrito que se presenta en forma de blog (con feedback en la sesión y en el blog).

Al ser blogs de trabajos académicos de alumnos, muchos grupos de estudiantes consideraron oportuno restringir el acceso a los mismos con una contraseña que facilitaban al profesor. Los siguientes blogs, que no tienen restricciones de acceso, pueden servir a modo de ejemplo: http://juniorfijo.blogspot.com/2011/05/introduccion-somos-alumnos-desegundo.html, http://juegosdeinvasion.wordpress.com y http://anbelmar.blogs.uv.es.

\section{d) Fundamentos de la expresión corporal}

La parte teórica de la evaluación continua de esta asignatura se compone de diez cuestiones que se deben responder a lo largo del curso y que obligan al alumno a relacionar los contenidos teóricos con las prácticas. Esta parte supone el $50 \%$ de la evaluación de la asignatura.

A lo largo del curso se ha utilizado el blog (http://expresioncorporal1011.blogspot.com) para articular cuatro cuestiones relacionadas con esta evaluación, lo que supone el $20 \%$ del total de la evaluación de la asignatura.

En las entradas del blog se han utilizado distintos recursos.

- En dos ocasiones para enlazar con recursos presentes en Youtube que debían ser vistos y analizados.

- Se propuso un enlace con la página Web del profesor para analizar un documento sonoro.

- En otra evaluación se planteaba una cuestión que debía ser debatida en grupo.

En todos los casos los análisis y las respuestas debían ser enviados al blog en forma de comentarios hasta una fecha determinada. Se utilizó la opción de moderación de comentarios por parte del profesor, por lo que los comentarios permanecían ocultos hasta la fecha establecida.

\section{e) La educación física como materia escolar}

La evaluación de esta asignatura se estructuró en dos bloques de actividad. El primero de ellos, englobó pruebas relacionadas con lecturas y actividades formativas realizadas total o parcialmente en el aula, que en su conjunto representaron el $65 \%$ de la calificación. El segundo bloque se refirió únicamente a un examen final de desarrollo, que supuso el 35\% 
restante. Esta prueba final fue voluntaria puesto que la asignatura podía superarse si se alcanzaba el $50 \%$ de la puntuación mediante las actividades del primer bloque.

El blog de la asignatura (http://efme2010.blogspot.com) se utilizó para llevar a cabo algunas de las actividades previstas en el primer bloque. La tarea planteada en la entrada formulada por el profesor era resuelta por los estudiantes durante su tiempo de trabajo autónomo y, a su vez, un grupo reducido de ellos (dos o tres) se encargó de revisar los comentarios y presentar una síntesis de los mismos en el aula, para iniciar la puesta en común y el análisis o discusión pertinente. Estas actividades tuvieron un valor del $15 \%$ de la calificación de la asignatura. Los estudiantes que se ofrecían para realizar el análisis previo y la síntesis tuvieron una gratificación adicional del 5\%.

\section{VALORACIÓN DE LA EXPERIENCIA Y CONCLUSIONES}

Aunque las formas de evaluar fueron distintas en función de la asignatura, en general los blogs sirvieron para poner una atención mayor en los procesos de construcción del aprendizaje y no solo en los resultados. Es decir, el uso de blogs ayudó a hacer visible ese proceso autónomo de resolución de las tareas y trabajos individuales o colectivos y la progresión de los aprendizajes. Específicamente, el peso de los porcentajes que se atribuyeron al trabajo realizado por los estudiantes, por su participación en el blog de cada asignatura, oscilaron entre el $15 \%$ y el $40 \%$.

Se constató que la integración de los blogs en sistemas de evaluación continua es una estrategia útil para reforzar el trabajo autónomo del alumnado. La redacción de los comentarios a los posts o las resoluciones de las tareas formuladas por los profesores a través de los blogs supusieron una oportunidad para profundizar en los contenidos de las asignaturas y para evaluar los aprendizajes. Al no estar sujetos al tiempo y al espacio del aula, los blogs incentivaron un nivel de reflexión mayor al del aula y la necesidad de documentarse respecto a las cuestiones planteadas. De esta manera, se estimularon capacidades personales de trabajo intelectual que favorecen una serie de competencias reclamadas en el EEES, como por ejemplo el aprender a aprender, o las vinculadas con el análisis y tratamiento de la información por parte del alumnado.

Los blogs constituyeron desde nuestra experiencia un archivo de las aportaciones de los estudiantes en relación con las actividades y el desarrollo de las asignaturas, que permitió al profesorado recabar información acerca de la comprensión de los contenidos y del progreso de los aprendizajes del alumnado. Utilizados así, los blogs fueron una herramienta fundamental para la evaluación continua, puesto que complementaron y enriquecieron los elementos de juicio que el profesorado consideraba en sus clases o a través de otras actividades de evaluación. La evaluación continua no se efectuó como una actividad específica, aparte y paralela al proceso de enseñanza, sino que formaba parte integrada de este proceso. Además de ser útil para calificar al alumnado, fue una evaluación consecuencia del esfuerzo docente por promover propuestas en las que el alumnado pueda aprender. Una evaluación que intentó ir más allá de la comprobación de la adquisición o recuerdo de información, preocupándose por ofrecer al estudiante un recurso que le hiciera consciente de las posibilidades y limitaciones de su propio aprendizaje.

El desarrollo de este proyecto supone una primera experiencia en el proceso de evaluación continua del aprendizaje del alumnado con el uso de blogs por parte del profesorado 
participante. Sabemos que la evaluación formativa y centrada en el aprendizaje del alumnado está muy relacionada con las implicaciones didácticas que tiene el proceso de convergencia hacia el denominado Sistema Europeo de Transferencia de Créditos (ECTS), por ello ha sido una de las principales preocupaciones el integrar los procesos de evaluación formativa a los más habituales de evaluación sumativa. Sin embargo, una de las dificultades detectadas fue compatibilizar las exigencias y la dedicación docente que requiere una evaluación formativa con el problema estructural de una elevada ratio alumnado-profesor (ver tabla 1). Es muy difícil hacer compatible una evaluación formativa con grupos tan masificados.

La principal contribución didáctica del proyecto desarrollado está en poner al alcance de la comunidad universitaria diversas posibilidades de evaluación continua del aprendizaje del alumnado en relación con el uso docente de los blogs. Esta experiencia ilustra diferentes estilos en el uso de blogs por parte de un conjunto de profesores que los utilizan, con distintas orientaciones, como herramienta para la evaluación continua, lo que pone de relieve diversas intenciones formativas y aspectos prácticos que pueden tenerse en cuenta para la valoración de las competencias $y$, especialmente, los contenidos genéricos transversales de los nuevos estudios de Grado.

Como conclusión, señalar que la evaluación continua vinculada con los blogs en la experiencia descrita supuso algo más que el uso de una tecnología. Tuvo que ver, fundamentalmente, con una actitud docente preocupada por compartir conocimientos con el alumnado, ampliar el aprendizaje más allá del aula a través de un espacio no presencial e implicar al alumnado en un aprendizaje autónomo y colaborativo. Estamos de acuerdo con Salinas (2004) en que los cambios de la Educación Superior relacionados con las TIC suponen, principalmente, cambios en el rol del profesor, cambios en el rol del alumnado y cambios metodológicos, pero como recogen Brown, Bull y Pendlebury (1997) los cambios metodológicos en la educación superior deben suponer también cambios en las formas de evaluación, porque sin cambios en la formas de evaluación, serán difíciles de conseguir los cambios metodológicos.

\section{Agradecimientos:}

Este trabajo procede del Proyecto de innovación educativa Evaluación continua del aprendizaje del alumnado en relación con el uso de blogs, financiado por la Universitat de València (convocatoria 2010-2011).

\section{REFERENCIAS BIBLIOGRÁFICAS}

AGUADED, J. I., LÓPEZ, E., ALONSO, L. (2010). Formación del profesorado y software social. Estudios sobre Educación, 18, 97-114. Recuperado el 9 de septiembre, 2012, de http://www.unav.es/educacion/ese/pagina 8.html

ANTOlín, L., MOLINA, P., VILLAMÓN, M., DEVÍS, J., PÉREZ, V. (2011). Uso de blogs en ciencias de la actividad física y el deporte. @tic. Revista d'innovació educativa, 7, 1218. Recuperado el 9 de septiembre, 2012, de http://ojs.uv.es/index.php/attic/article/download/261/1015 
BOHÓRQUEZ, E. (2008). El blog como recurso educativo. Edutec, Revista Electrónica de Tecnología Educativa, 40. Recuperado el 11 de septiembre, 2012, de http://edutec.rediris.es/Revelec2/Revelec26/articulos n26 PDF/Edutec-

E Bohorquez_n26-\%203.pdf

BLANCO, S. (2005). Los weblogs como herramienta didáctica en el seno de una asignatura curricular. En: G. García (Coord.). El ecosistema digital: modelos de comunicación, nuevos medios y público en Internet (pp. 151-166). Valencia: Servei de Publicacions de la Universitat de València. Recuperado el 9 de septiembre, 2012, de http://www.vinv.ucr.ac.cr/docs/divulgacion-ciencia/libros-y-tesis/ecosistemadigital.pdf

BROWN, G., BULL, J. Y PENDLEBURY, M. (1997). Assessing Student Learning in Higher Education. Londres: Routledge.

CHURCHILL, D. (2009). Educational applications of Web 2.0: Using blogs to support teaching and learning. British Journal of Educational Technology, 40(1), 179-183. Recuperado el 11 de septiembre, 2012, de http://onlinelibrary.wiley.com/doi/10.1111/i.14678535.2008.00865.x/full

DUCATE, L.C. Y LOMICKA, L.L. (2008). Adventures in the blogosphere: From blog readers to blog writers. Computer Assisted Language Learning, 21(1), 9-28. Recuperado el 4 de septiembre, 2012, de http://pdfserve.informaworld.com/408796 790622329.pdf

FARMER, B., YUE, A. Y BROOKS, C. (2008). Using blogging for higher order learning in large cohort university teaching: A case study. Australasian Journal of Educational Technology, 24(2), 123-136. Recuperado el 11 de septiembre, 2012, de http://www.ascilite.org.au/ajet/ajet24/farmer.html

FERREYRO, J. (2007). Abriendo el aula. Blogs, una reflexión compartida sobre buenas experiencias de enseñanza. EDUTEC, Revista Electrónica de Tecnología Educativa, 40. Recuperado el 4 de septiembre, 2012, de http://edutec.rediris.es/Revelec2/revelec24/ifereyro/iferreyro.html

FRAILE, A. (2002). El seminario colaborativo: una propuesta formativa para el profesorado de Educación Física. Contextos educativos: Revista de educación, 5, 101-122.

GEWERC, A. (2005). El uso de weblogs en la docencia universitaria. Revista Latinoamericana de Tecnología Educativa, 4(1), 9-23. Recuperado el 9 de septiembre, 2012, de http://campusvirtual.unex.es/cala/editio/index.php?journal=relatec\&page=article\&o p=view\&path[]=173\&path[]=163

GLOGOFF, S. (2005). Instructional Blogging: Promoting Interactivity, Student- Centered Learning, and Peer Input. Innovate, 1(5). Recuperado el 4 de septiembre, 2012, de http://www.innovateonline.info/index.php?view=article\&id=126

GONZÁLEZ, R. Y GARCÍA, F.E. (2009). El blog en la docencia universitaria ¿una herramienta útil para la convergencia europea? Relada, 3(2), 135-144. Recuperado el 11 de septiembre, 2012, de http://serviciosgate.upm.es/ojs/index.php/relada/article/viewFile/70/70 
LADYSHEWSKY, R.K. Y GARDNER, P. (2008). Peer assisted learning and blogging: A strategy to promote reflective practice during clinical fieldwork. Australasian Journal of Educational Technology, 24(3), 241-257. Recuperado el 11 de septiembre, 2012, de http://www.ascilite.org.au/ajet/ajet24/ladyshewsky.html

LORENZO, M., TRUJILLO, J. M., LORENZO, R. Y PÉREZ, E. (2011). Usos del Weblog en la universidad para la gestión de conocimiento y trabajo en red. Pixel-bit. Revista de Medios y Educación, 39, 141-154. Recuperado el 8 de septiembre, 2012, de http://intra.sav.us.es:8080/pixelbit/images/stories/p39/11.pdf

MARZAL, M.Á. Y BUTERA, M.J. (2007). Los blogs en el nuevo modelo educativo universitario: posibilidades e iniciativas. BID: textos universitaris de biblioteconomia $i$ documentació, 19. Recuperado el 14 de septiembre, 2012, de http://www.ub.es/bid/19marza2.htm

ORIHUELA, J.L. Y SANTOS, M.L. (2004). Los weblogs como herramienta educativa: Experiencias con bitácoras de alumnos. Quaderns Digitals net, 34. Recuperado el 4 de septiembre, 2012, de http://www.quadernsdigitals.net/index.php?accionMenu=hemeroteca.VisualizaArtic ulolU.visualiza\&articulo id=7751

PÉREZ, F., ARANDA, E., HERNÁNDEZ, A., MARTíN, A., BENITO, M.J. Y CóRdOBA, M. (2012). Otras actividades complementarias para mejorar la docencia universitaria: Elaboración y utilización de blogs. Edutec, Revista Electrónica de Tecnología Educativa, 40. Recuperado el 3 de octubre, 2012, de http://edutec.rediris.es/Revelec2/Revelec40/actividades complementarias mejora docencia universitaria elaboracion y utilizacion blogs.html

PHILIP, R. Y NICHOLLS, J. (2009). Group blogs: Documenting collaborative drama processes. Australasian Journal of Educational Technology, 25(5), 683-699. Recuperado el 9 de septiembre, 2012, de http://www.ascilite.org.au/ajet/ajet25/philip.html

SALINAS, J. (2004). Innovación docente y uso de las TIC en la enseñanza universitaria. Revista Universidad y Sociedad del Conocimiento, 1(1). Recuperado el 3 de octubre, 2012, de http://rusc.uoc.edu/ojs/index.php/rusc/article/view/228

SALINAS, M.I. Y VITICCIOLI, S.M. (2008) Innovar con blogs en la enseñanza universitaria presencial. Edutec, Revista Electrónica de Tecnología Educativa, 40. Recuperado el 11 de septiembre, 2012, de http://edutec.rediris.es/Revelec2/revelec27/articulos n27 PDF/EdutecE MISanilas Viticcioli $\mathrm{n} 27 . \mathrm{pdf}$

SANTOVEÑA, S. M. (2011). Análisis Socioeducativo blogueo como actividad alfabetizadota y colaborativa. Edutec, Revista Electrónica de Tecnología Educativa, 40. Recuperado el 2 de septiembre, 2012, de http://edutec.rediris.es/Revelec2/Revelec35/pdf/Edutece n35 Santovena.pdf

SHIM, J.P. Y GUO, C. (2009). Weblog technology for instruction, learning, and information delivery. Decision Sciences Journal of Innovative Education, 7(1), 171-193. Recuperado el 4 de septiembre, 2012, de http://onlinelibrary.wiley.com/doi/10.1111/j.1540-4609.2008.00212.x/full 
TORRES, V. (2009). ¿Por qué las bitácoras electrónicas (blogs) se usan poco para estudiar ciencias físico-matemáticas? Edutec, Revista Electrónica de Tecnología Educativa, 40. Recuperado el 4 de octubre, 2012, de http://edutec.rediris.es/Revelec2/revelec29/articulos n29 pdf/6EdutecE Torres n29.pdf

WILLIAMS, J.B. Y JACOBS, J. (2004). Exploring the use of blogs as learning spaces in the higher education sector. Australasian Journal of Educational Technology, 20(2), 232-247. Recuperado el 4 de septiembre, 2012, de http://www.ascilite.org.au/ajet/ajet20/williams.html

XIE, Y., KE, F. Y SHARMA, P. (2008). The effect of peer feedback for blogging on college students' reflective learning processes. Internet and Higher Education, 11(1), 18-25. Recuperado el 4 de septiembre, 2012, de http://www.sciencedirect.com/science/article/pii/S1096751607000693

Para citar este artículo:

PERE MOLINA, P., ANTOLÍN JIMENO, L., PÉREZ-SAMANIEGO, V., DEVIS-DEVIS, J., VILLAMÓN, M. \& VALENCIANO VALCÁRCEL, J. (2013). Uso de blogs y evaluación continua del aprendizaje del alumnado universitario. EDUTEC, Revista Electrónica de Tecnología Educativa, 43 Recuperado

el $\mathrm{dd} / \mathrm{mm} / \mathrm{aa}$

de

http://edutec.rediris.es/Revelec2/Revelec43/blogs evaluacion aprendizaje alumnado univ ersitario.html 